\title{
Observation of an Anomalous Number of Dimuon Events in a High Energy Neutrino Beam
}

\author{
T. Adams ${ }^{4}$, A. Alton ${ }^{4}$, S. Avvakumov ${ }^{8}$, L. de Barbaro ${ }^{5}$, P. de Barbaro ${ }^{8}$, R. H. Bernstein ${ }^{3}$, A. Bodek ${ }^{8}$, T. Bolton ${ }^{4}$,

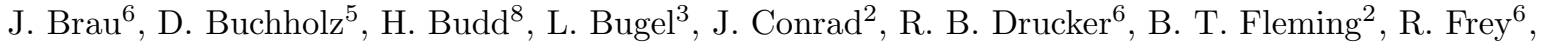 \\ J.A. Formaggio ${ }^{2}$, J. Goldman ${ }^{4}$, M. Goncharov ${ }^{4}$, D. A. Harris ${ }^{8}$, R. A. Johnson ${ }^{1}$, J. H. Kim² , S. Koutsoliotas ${ }^{2}$, \\ M. J. Lamm ${ }^{3}$, W. Marsh ${ }^{3}$, D. Mason ${ }^{6}$, J. McDonald ${ }^{7}$, C. McNulty ${ }^{2}$, K. S. McFarland ${ }^{3}$, D. Naples ${ }^{7}$, P. Nienaber ${ }^{3}$, \\ A. Romosan ${ }^{2}$, W. K. Sakumoto ${ }^{8}$, H. Schellman ${ }^{5}$, M. H. Shaevitz ${ }^{2}$, P. Spentzouris ${ }^{2}$, E. G. Stern ${ }^{2}$, N. Suwonjandee ${ }^{1}$, \\ M. Tzanov ${ }^{7}$, M. Vakili ${ }^{1}$, A. Vaitaitis ${ }^{2}$, U. K. Yang ${ }^{8}$, J. Yu$^{3}$, G. P. Zeller ${ }^{5}$, and E. D. Zimmerman ${ }^{2}$ \\ ${ }^{1}$ University of Cincinnati, Cincinnati, OH 45221 \\ ${ }^{2}$ Columbia University, New York, NY 10027 \\ ${ }^{3}$ Fermi National Accelerator Laboratory, Batavia, IL 60510 \\ ${ }^{4}$ Kansas State University, Manhattan, KS 66506 \\ ${ }^{5}$ Northwestern University, Evanston, IL 60208 \\ ${ }^{6}$ University of Oregon, Eugene, OR 97403 \\ ${ }^{7}$ University of Pittsburgh, Pittsburgh, PA 15260 \\ ${ }^{8}$ University of Rochester, Rochester, NY 14627
}

(October 31, 2018)

\begin{abstract}
A search for long-lived neutral particles $\left(N^{0}\right.$ s) with masses above $2.2 \mathrm{GeV} / c^{2}$ that decay into at least one muon has been performed using an instrumented decay channel at the $\mathrm{NuTeV}$ experiment at Fermilab. Data were examined for particles decaying into the final states $\mu \mu, \mu e$, and $\mu \pi$. Three $\mu \mu$ events were observed over an expected Standard Model background of $0.069 \pm 0.010$ events; no events were observed in the other modes.
\end{abstract}

PACS numbers: 14.80.-j, 13.85.Rm, 12.60.Jv, 13.15+g

A number of extensions to the Standard Model (SM) of elementary particle physics predict new particles with reduced couplings to normal quarks and leptons. Some of these particles, which we shall refer to as $N^{0}$ 's, may have zero electric charge, long lifetimes, and small interaction rates with ordinary matter. Examples include neutral heavy leptons (NHLs) or heavy sterile neutrinos [1 [3] and neutral supersymmetric particles [4]. The $N^{0}$ may be either pair-produced in hadronic interactions or occur as a daughter particle in weak decays of mesons through mixing with SM neutrinos. Decays of the $N^{0}$ can proceed through $W^{ \pm}$or $Z^{0}$ mediated decays via mixing, or through $R$-parity violating supersymmetric processes. We report here the results of a search for $N^{0}$ particles in the mass region above $2.2 \mathrm{GeV} / c^{2}$ that decay into final states with at least one muon and one other charged particle, using the NuTeV detector at Fermilab (E815). This analysis extends our results for a previous search for $N^{0}$ 's in the mass region 0.3 to $3.0 \mathrm{GeV} / c^{2}$ with at least one final state muon [5], and for a search for $N^{0}$ 's with mass $\leq 0.3 \mathrm{GeV} / c^{2}$ that decay to $e^{+} e^{-} \nu$ [6].

$\mathrm{NuTeV}$ received $2.54 \times 10^{18} 800 \mathrm{GeV} / c$ protons during the 1997 fixed-target run at Fermilab with the detector configured for this search. The proton beam was incident on a one-interaction-length beryllium oxide target at a targeting angle of $7.8 \mathrm{mr}$ with respect to the detector. A sign-selected quadrupole train (SSQT) [7] focused either positive ( $45 \%$ of the running) or negative (55\% of the running) secondary $\pi$ and $K$ mesons into a $440 \mathrm{~m}$ evacuated pipe pointed toward the $\mathrm{NuTeV}$ experiment. Surviving neutrinos, and possibly $N^{0}$ 's, traversed $\sim 850$ meters of earth-steel shielding before reaching the $\mathrm{NuTeV}$ decay channel.

The decay channel region (Fig. 11), located 1.4 $\mathrm{km}$ downstream of the production target, was designed to search for exotic neutral particle decays in a $(1.27 \times 1.27 \times 34) \mathrm{m}^{3}$ fiducial volume. The volume consisted of large helium-filled plastic bags interspersed with drift chambers (DC). An array of plastic scintillation counters at the upstream end of the decay channel provided a means to veto charged particles produced in the upstream shielding. The Lab E neutrino detector [8,9] downstream of the decay channel provided final state particle energy measurement and identification. This detector consisted of a 690 ton iron-scintillator sampling calorimeter, instrumented with DC for charged particle tracking, followed by a toroid muon spectrometer. Sets of hits in the calorimeter DC were linked to tracks found with the decay channel DC system to determine particle identification. Muons were identified by penetration, electrons by their characteristic short clusters of hits, and charged hadrons by their elongated hit patterns. All charged hadrons were assumed to be pions. The particle identification algorithm was tuned with electrons, pions, 
and muons provided by the $\mathrm{NuTeV}$ calibration beam [9]. The probability to mis-reconstruct a dimuon event as a $\mu e$ or $\mu \pi$ event was $0.2 \% ; \mu e$ and $\mu \pi$ events were misreconstructed as $\mu \mu$ events with a probability of $0.4 \%$ and $1.3 \%$ respectively. Muon energy was determined by either spectrometer measurement $\left(\sigma_{p} / p=11 \%\right)$, range in the calorimeter $\left(\sigma_{p}=310 \mathrm{MeV}\right)$, or multiple scattering $\left(\sigma_{p} / p=42 \%\right.$ at $\left.p=50 \mathrm{GeV} / c\right)$. Only muons penetrating to the toroid spectrometer could be charge-identified. The hadronic energy resolution of the calorimeter was $\sigma / E=(0.024 \pm 0.001) \oplus(0.874 \pm 0.003) / \sqrt{E(\mathrm{GeV})}$; the electromagnetic energy resolution, $\sigma / E=(0.04 \pm 0.001) \oplus$ $(0.52 \pm 0.01) / \sqrt{E(\mathrm{GeV})}[9]$.

Event selection criteria were developed to minimize known backgrounds while maintaining efficiency for a possible $N^{0}$ signal. A series of cuts isolated events with exactly two well-reconstructed tracks forming a vertex within the decay channel fiducial volume and having no charged particle identified in the upstream veto system. Both tracks were required to be well-reconstructed and have an associated calorimeter cluster, with at least one of the tracks identified as a muon. The track and vertex quality criteria were numerically the same as in Ref. [5]. The probability for a signal event to fail this is $<2 \%$. The vertex position was required to be within the detector fiducial volume; in addition, the longitudinal distance from the vertex position to any drift chamber was required to be greater than the larger of $101.6 \mathrm{~cm}$ and $3 \sigma_{z}$, with $\sigma_{z}$ the longitudinal vertex position error. A third track which formed a downstream vertex with one of the two initial tracks was permitted, to allow for $\delta$ ray emission. Cosmic ray tracks (which are suppressed by the fast gate timing of the neutrino beam) were removed by requiring the slope of each track relative to the beam direction be less than $100 \mathrm{mr}$. Muons, hadrons, and electrons were required to have an energy greater than $2.2 \mathrm{GeV}, 10 \mathrm{GeV}$, and $10 \mathrm{GeV}$, respectively, with an additional total energy cut of $12 \mathrm{GeV}$ applied to $\mu \mu$ events. In order to isolate high mass events, a transverse mass cut $m_{T}>2.2 \mathrm{GeV} / c^{2}$ was applied, with $m_{T} \equiv\left|P_{T}\right|+\sqrt{P_{T}^{2}+m_{V}^{2}}, P_{T}$ the component of the total reconstructed momentum perpendicular to the beam direction, and $m_{V}$ the invariant mass of the visible particles.

Further "clean event" cuts were applied to reduce the level of the dominant deep-inelastic neutrino scattering (DIS) backgrounds. DIS events typically had large track multiplicities, many drift chamber hits, and extra unassociated clusters in the calorimeter. Clean cuts required: (1) three or fewer tracks in any one DC view, (2) three or fewer DC hits in any view of the first chamber downstream of the vertex, (3) at least one DC view with fewer than eight hits total in the first two chambers downstream of the vertex, (4) no energy clusters in the calorimeter not associated with tracks, and (5) no tracks identified as electrons with missing hits in either view of the first two chambers downstream of the vertex. The final cut removed events where a photon from the primary vertex converted to $e^{+} e^{-}$and was reconstructed as an electron.

Detailed MC simulations of physics processes including detector effects were used to estimate possible backgrounds to the $N^{0}$ signal. Two major classes of physics processes considered included DIS [10], resonance production [11], and diffractive scattering [12] by neutrinos; and decays and interactions of hadrons and photons produced in neutrino interactions. Particular attention was given to known sources of dimuon or dimuon-like production: DIS, resonance, and diffractive production of charm; neutrino trident production; $\mu^{+} \mu^{-}$vector meson decays; electromagnetic muon pair production; low multiplicity $\nu_{\mu}$ DIS accompanied by a secondary pion or kaon decay; and decays of $K_{L}^{0}$ mesons produced by neutrino interactions in the decay channel or surrounding material. Approximately $10^{3}$ times as many neutrino interactions as in the data were simulated in the decay channel volume; in addition, a large sample of DIS events was generated in the material surrounding the decay channel. Event generators fed a GEANT-based [13] detector simulation that produced hit-level simulations of raw data including DC inefficiencies and noise. MC events were processed using the same analysis routines used for the data. A number of other possible sources were studied as well and found to be negligible.

Background calculations were normalized to data using charged-current DIS interactions in the decay channel DC. Events in this sample were required to pass the following five normalization cuts: a vertex within the transverse fiducial volume; a $z$ vertex within $76.2 \mathrm{~cm}$ of a DC; no upstream veto; $\geq 1 \mathrm{GeV}$ energy deposit in the front of the calorimeter; and one toroid-analyzed muon matched to a decay channel track. The MC was normalized to match the total number of data events with two or more tracks; the error on this normalization is 9\%. Figure 2 shows a comparison of the longitudinal vertex resolution between data and $\mathrm{MC}$ with the $z$ vertex requirement removed.

$\mathrm{MC}$ events were also compared to another data control sample as a check on the quality of the simulation. For this sample, the vertex was required to be within the decay channel transverse fiducial volume but the $z$ position was allowed to be either in the chambers or the helium. Tight track angle cuts were imposed, and there was a strict requirement on veto system activity. The majority of these events were from interactions in the chamber material or from interactions in the laboratory floor. Of 495 
events in the data, 116 had vertices reconstructed in the helium at least $101.6 \mathrm{~cm}$ from the nearest DC. This can be compared to the MC, which predicted $(514 \pm 82)$ total events and $(96 \pm 15)$ events reconstructed in the helium. Because loose vertex quality requirements allowed misreconstructed interactions in the chambers and floor to enter this sample, only $35 \%$ of the vertices reconstructed in the helium were actually due to $\nu$-He interactions.

After all cuts, the expected background to the $N^{0}$ search was $0.069 \pm 0.010$ events in $\mu \mu$ mode, $0.13 \pm 0.02$ events in $\mu e$ mode and $0.14 \pm 0.02$ events in $\mu \pi$ mode. Table If summarizes the background components for $\mu \mu$ mode; DIS clearly dominates.

Before looking at the data in the signal region, we performed a series of analyses on other fiducial and kinematic ranges. These included using: (1) identical analysis cuts applied to events within $15.2 \mathrm{~cm}$ of a DC (the "chamber region"); (2) the chamber region with loosened cuts to increase $\mu \pi$ acceptance; (3) the "intermediate region" between 15.2 and $101.6 \mathrm{~cm}$ from the chambers, with otherwise standard analysis cuts; and (4) events with well-reconstructed two-track vertices where the tracks were both identified as pions. Measurements agreed with MC predictions within $1.5 \sigma$ in all cases. For example in the "chamber region" sample (1), two two-track events were observed in the data with 3.5 predicted by the MC.

Upon examining the signal region, three $\mu \mu(\nu)$ events were observed, which is considerably above the predicted background. No $\mu e$ or $\mu \pi$ events were observed, which is consistent with background estimates. Table II summarizes event reconstruction characteristics of the $\mu \mu$ events. The vertex and track probabilities have been measured with both the signal and background Monte Carlos and represent the probability that the vertex (or track) would reconstruct with a similar (or worse) quality.

Observation of three $\mu \mu$ data events prompted further tests comparing data to $\mathrm{MC}$ predictions with relaxed cuts. These included gradually removing the cuts and alternately releasing and then returning individual cuts and cut-pairs. These studies revealed no significant discrepancy between data and the background calculations (except for the original $3 \mu \mu$ events).

The three $\mu \mu$ events have some features consistent with a $N^{0}$ decay hypothesis. The events pass the analysis cuts, where the background is estimated to be 0.069 events. All three occur well within the fiducial volume away from the chambers and are evenly distributed throughout the decay channel. The transverse mass, invariant mass, and missing $P_{T}$ are all consistent with the decay of an $N^{0}$ with a mass of about $5 \mathrm{GeV} / c^{2}$. Since only $1(0) \mu e$ and $2(0) \mu \pi$ events (consistent with MC expectations) were observed in the chamber (helium) data, it is unlikely that $\mu \mu$ events are related to low multiplicity neutrino events followed by $\pi$ and $K$ decay.

Unlike the background, in both an NHL or neutralino model one would expect the $\mu \pi$ rate to be highly suppressed relative to leptonic decays. However, for a 5 $\mathrm{GeV} / c^{2}$ NHL model, one would expect 1.4 times more $\mu e \nu$ events [3]. A neutralino model, on the other hand, can accommodate the observation of either only $\mu \mu$ or a combination of $\mu \mu$ and $\mu e$ candidates by selecting appropriate couplings.

However, several aspects of the candidate events are similar to those from neutrino interaction backgrounds, and might be indicative of unaccounted-for sources or a statistical fluctuation. Globally, the events share one feature that is improbable for an $N^{0}$ decay hypothesis. All three events have a muon energy asymmetry $A>0.83$, where $A \equiv\left(\left|E_{1}-E_{2}\right|\right) /\left(E_{1}+E_{2}\right)$. For DIS background, the probability for three $\mu \mu$ events which pass the signal cuts to have the observed energy asymmetry is $38 \%$. The probability that this occurs in a weak decay hypothesis [14] is less than $0.5 \%$ (including acceptance). All three events occurred during the higher rate $\nu$-mode rather than $\bar{\nu}$-mode running periods. In the two events where the charge of the higher-momentum muon can be measured, it has the same sign as expected for the leading muon in a charged-current neutrino interaction. Event kinematics $\left(M_{T}, M_{\mu \mu}, P_{T}\right)$ are also consistent with DIS characteristics. However, the observed number of events is inconsistent with expected neutrino interaction background.

In summary, $\mathrm{NuTeV}$ has observed three $\mu \mu$ events, zero $\mu \pi$, and zero $\mu e$ events with transverse mass above 2.2 $\mathrm{GeV} / c^{2}$. The expected backgrounds were $0.069 \pm 0.010$, $0.14 \pm 0.02$, and $0.13 \pm 0.02$ events, respectively. The rate corresponding to the observed three events is not consistent with Standard Model processes we have identified and the source of the events is not clear. NuTeV is insensitive to NHL production in this mass region, but can set an interesting limit on neutralino production (determined by calculating one-sided limits using a frequentist approach without background subtraction [15]). NuTeV is the first experiment to set limits on the production of long-lived neutralinos in this mass range which decay by $R$-parity violation. This limit (Fig. 3), although motivated by a neutralino hypothesis, is a generic limit applicable for any model of neutral particle production at the target 4 .

This research was supported by the U.S. Department of Energy and the National Science Foundation. We thank the staff of FNAL for their contributions to the construction and support of this experiment during the 1996-97 fixed target run. 


\section{REFERENCES}

[1] M. Gronau, C. N. Leung, and J. L. Rosner, Phys. Rev. D 29, 2539 (1984).

[2] R. E. Shrock, Phys. Rev. D 24, 1232 (1981).

[3] L. M. Johnson, D. W. McKay, and T. Bolton, Phys. Rev. D 56, 2970 (1997).

[4] L. Borissov et al., hep-ph0007195.

[5] A. Vaitaitis et al., Phys. Rev. Lett. 83, 4943 (1999).

[6] J. A. Formaggio et al., Phys. Rev. Lett. 84, 4043 (2000).

[7] J. Yu et al., "Technical Memorandum: NuTeV SSQT performance," Report No. FERMILAB-TM-2040, 1998.

[8] W. Sakumoto et al., Nucl. Instrum. Methods A 294, 179 (1990); B. King et al., Nucl. Instrum. Methods A 302, 254 (1991).

[9] D. Harris et al., Nucl. Instrum. Methods A 447, 377 (2000).

[10] W. G. Seligman et al., Phys. Rev. Lett. 79, 1213 (1997).

[11] R. Belusevic and D Rein, Phys. Rev. D 38, 2753 (1988).

[12] T. Adams et al., Phys. Rev. D 61, 092001 (2000).

[13] Applications and Software Group, CERN, "GEANT: Detector Description and Simulation Tool," CERN Program Library Report Q123.

[14] J. A. Formaggio et al., Phys. Rev. D 57, 7037 (1998).

[15] G. Feldman and R. Cousins, Phys. Rev. D 57, 3873 (1998).

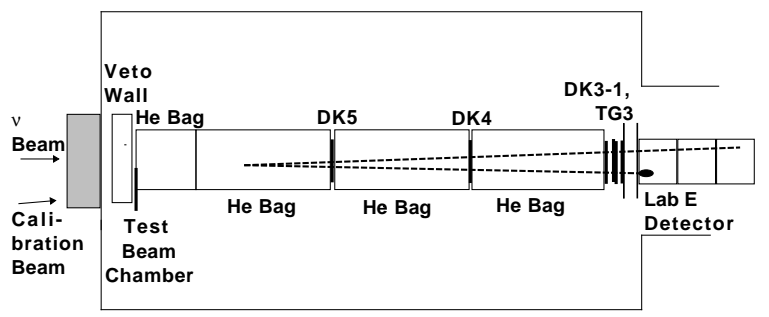

FIG. 1. Schematic of the NuTeV decay channel with example $N^{0} \rightarrow \mu \pi$ decay.

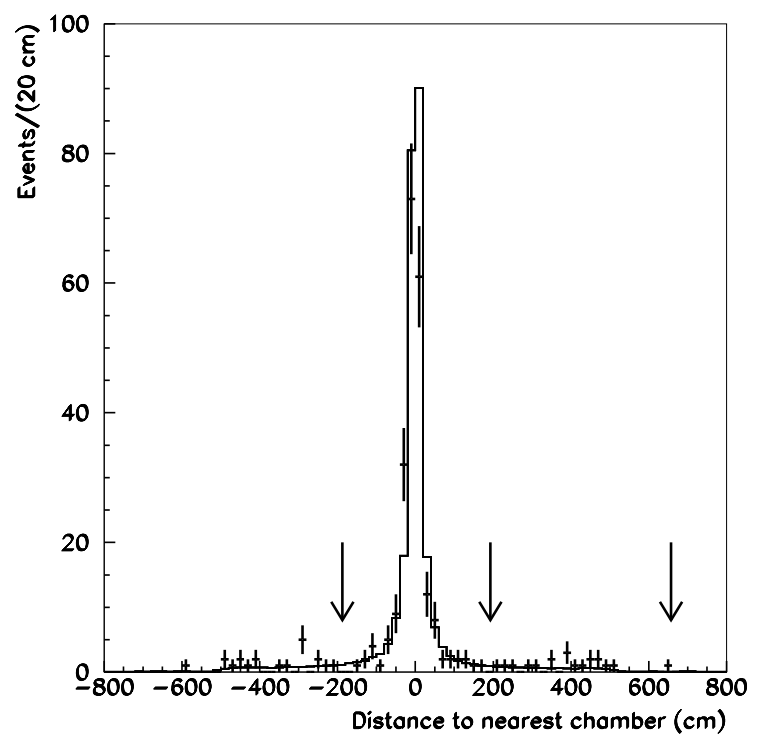

FIG. 2. Distance of longitudinal vertex position from the closest chamber for events in the normalization sample without the $z$ vertex requirement. (Crosses: data; histogram: MC.; arrows: values for the three observed $\mu \mu$ events)

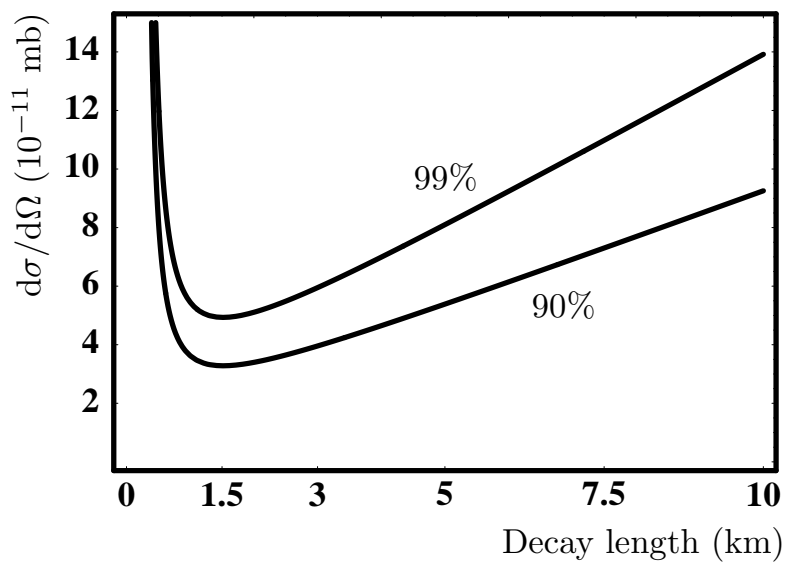

FIG. 3. NuTeV limit on neutralino production. The plot gives the limit on the differential p-p cross section to produce an $N^{0}$ versus the decay length of the $N^{0}$ in the laboratory coordinate system. This limit is generic for an $N^{0}$ produced at the target. 
TABLE I. Estimated rates of background to the $N^{0} \rightarrow \mu \mu(\nu)$ search

\begin{tabular}{lc}
\hline \hline Source & $\mu \mu(\nu)$ events \\
\hline DIS events & $(6.8 \pm 1.0) \times 10^{-2}$ \\
Diffractive charm & $(1.3 \pm 0.1) \times 10^{-3}$ \\
Diffractive $\pi$ & $(1.9 \pm 0.1) \times 10^{-4}$ \\
Diffractive $K$ & $(4.0 \pm 0.3) \times 10^{-7}$ \\
$\mathrm{~K}_{L}^{0}$ decays from shielding & $(3.9 \pm 3.9) \times 10^{-4}$ \\
Other sources & $\ll 2.5 \times 10^{-4}$ \\
\hline Total $\mu \mu(\nu)$ Background & $(6.9 \pm 1.0) \times 10^{-2}$ \\
\hline \hline
\end{tabular}

TABLE II. Kinematic and reconstruction quantities for the three candidate $N^{0} \rightarrow \mu \mu(\nu)$ events. The variables refer to the muons energies $\left(\mathrm{E}_{\mu 1}, \mathrm{E}_{\mu 2}\right)$, missing transverse momentum $\left(P_{T \text { miss }}\right)$, two muon invariant mass $\left(m_{\text {inv }}\right)$, transverse mass $\left(m_{T}\right.$, see text $)$, transverse vertex position $\left(v_{x}, v_{y}\right)$, longitudinal distance to nearest drift chamber $(|\Delta z|)$, vertex probability $\left(\mathcal{P}_{\text {vert }}\right)$, first muon track probability $\left(\mathcal{P}_{\mu 1}\right)$, and second muon track probability $\left(\mathcal{P}_{\mu 2}\right)$. The probabilities are measured the with signal(background) Monte Carlos. The sign on the muon energy refers to the charge of the muon (if measured).

\begin{tabular}{cccc}
\hline \hline Event & 1 & 2 & 3 \\
\hline $\mathrm{E}_{\mu 1}(\mathrm{GeV})$ & -77.4 & -92.0 & \pm 48.0 \\
$\mathrm{E}_{\mu 2}(\mathrm{GeV})$ & \pm 2.56 & \pm 5.85 & \pm 4.34 \\
$P_{T \text { miss }}(\mathrm{GeV} / c)$ & 2.42 & 1.41 & 2.07 \\
$m_{\text {inv }}\left(\mathrm{GeV} / c^{2}\right)$ & 1.10 & 0.88 & 3.57 \\
$m_{T}\left(\mathrm{GeV} / c^{2}\right)$ & 5.08 & 3.08 & 4.66 \\
$v_{x}(\mathrm{~cm})$ & -46.5 & 48.0 & -57.5 \\
$v_{y}(\mathrm{~cm})$ & 3.4 & -38.3 & 15.0 \\
$|\Delta z|(\mathrm{cm})$ & 193 & 657 & -188 \\
$\left(\mathcal{P}_{\text {vert }}\right)$ & $0.81(0.94)$ & $0.043(0.48)$ & $0.011(0.30)$ \\
$\left(\mathcal{P}_{\mu 1}\right)$ & $0.95(0.96)$ & $0.034(0.18)$ & $0.51(0.73)$ \\
$\left(\mathcal{P}_{\mu 2}\right)$ & $0.49(0.70)$ & $0.034(0.18)$ & $0.15(0.39)$ \\
\hline \hline
\end{tabular}

